\title{
Commentary: Bone density in women with inflammatory bowel disease
}

\author{
A Hillary Steinhart MD MSc FRCPC
}

\begin{abstract}
ARTICLE
Bernstein CN, Leslie WD, Taback SP. Bone density in a population-based cohort of premenopausal adult women with early onset inflammatory bowel disease. Am J Gastroenterol 2003;98:1094-100.
\end{abstract}

\begin{abstract}
ARTICLE SUMMARY
Conventional wisdom suggests that children who develop inflammatory bowel disease are at particularly increased risk of osteopenia and osteoporosis because of the effects of disease activity, nutritional factors and medications, especially glucocorticoids, during critical periods of bone growth. In this study, Bernstein et al endeavoured to determine the prevalence of reduced bone mineral density (BMD) in a population-based cohort of women with onset of inflammatory bowel disease (IBD) before 20 years of age. A secondary goal of the study was to compare estimates of the rates of osteopenia and osteoporosis using two different techniques: the more conventional areal $\mathrm{BMD}$ and volumetric BMD. The latter measure is designed to account for the reduction in bone size that can occur because of growth delay associated with childhood IBD. On the other hand, areal BMD is said to underestimate true bone density. The authors identified a cohort of patients using their research registry of IBD patients in Manitoba. Women who developed IBD before 20 years of age and who were younger than age 45 at the time of the study were invited to complete a questionnaire and undergo dual energy x-ray absorptiometry (DEXA). Of the 148 subjects who were approached, 70 were eligible and agreed to participate. More than $80 \%$ of the participating subjects had Crohn's disease (CD) and more than $80 \%$ affected had onset of disease after puberty. BMD did not appear to differ between the patients with prepubertal and those with postpubertal onset. Twenty-five of the 70 subjects had an areal T score less than -1 at one or more site. Only three had osteoporosis ( $\mathrm{T}$ score less than -2.5$)$. Individuals with a $T$ score less than -1 had lower body weight and were more likely than other patients to have experienced amenorrhea in the year prior to DEXA testing. When $\mathrm{T}$ scores were adjusted for abnormalities in bone size, the
\end{abstract}

mean volumetric $\mathrm{T}$ score was slightly but statistically significantly higher than the corresponding areal $\mathrm{T}$ score at each site.

\section{COMMENTARY}

With this study, Bernstein et al have again made an important contribution to our understanding of the loss of bone density in patients with IBD. Their use of a population-based research registry in the province of Manitoba has been a major strength of this and previous studies $(1,2)$. Their finding of a $35.7 \%$ prevalence of osteopenia ( $\mathrm{T}$ score less than -1 at one or more site) is in keeping with previous studies, but the $4.2 \%$ prevalence of osteoporosis is at the lower end of the range found in other studies, particularly those including mostly or exclusively CD patients (3-5). This low prevalence of osteoporosis may reflect the fact that this study uses a population-based cohort, whereas previous studies involved cohorts of patients from tertiary care centers who might have been at increased risk of osteoporosis because they had more severe or complicated IBD.

Although the cohort in Bernstein et al's study more closely reflected the target population than did previous study populations, there are two areas to consider. Firstly, fewer than half of the eligible subjects actually agreed to participate in the study, complete the questionnaire and undergo DEXA. Secondly, nonparticipants differed in important ways from those who agreed to be studied. Although some of these differences, such as the higher proportion affected with ulcerative colitis and the younger ages of nonparticipating patients, might have led to an overestimation of the prevalence of osteopenia and osteoporosis in the target population, it is difficult to know what factors led to their nonparticipation and what effects these might have had on the prevalence estimates. Although these issues do not necessarily invalidate the study's conclusions, they are 'red flags' suggesting that the issue may not yet be settled.

The authors also conclude that the onset of disease before puberty per se does not increase the risk of osteoporosis or osteopenia. However, the power of the study to detect such an increase in risk was limited by the small number of prepubertal onset patients who were studied. Amenorrhea appeared to be a risk factor for diminished BMD. This finding underscores the importance of assessing the menstrual history in women 
patients with IBD, since reduced sex hormone secretion interferes with bone formation (6). Nevertheless, it should be remembered that a causal association is not proven by a retrospective study of this type. For example, it is possible that amenorrhea is a marker of other risk factors, such as increased disease severity or diminished body mass index, which was also found to correlate with decreased BMD in this and other studies (6).

Another important issue raised by the authors is the applicability of areal BMD measurements in individuals who have

\section{REFERENCES}

1. Bernstein CN, Blanchard JF, Leslie W, Wajda A, Yu BN. The incidence of fracture among patients with inflammatory bowel disease. A population-based cohort study. Ann Intern Med 2000;133:795-9.

2. Bernstein CN, Blanchard JF, Metge C, Yogendran M. The association between corticosteroid use and development of fractures among IBD patients in a population-based database. Am J Gastroenterol 2003;98:1797-801.

3. Andreassen H, Hylander E, Rix M. Gender, age, and body weight are the major predictive factors for bone mineral density in Crohn's experienced reduced bone growth associated with short stature. This may be particularly relevant to individuals who experience onset of IBD before the completion of their linear growth. The authors demonstrated that the use of volumetric BMD results in apparent 'improvement' in T scores at all sites measured. Unfortunately, there are no outcome data relating volumetric BMD measurements to fracture risk to help put this finding in perspective. Regardless, this study sheds further light on a potentially important issue that merits additional investigation.

disease: A case-control cross-sectional study of 113 patients. Am J Gastroenterol 1999;94:824-8.

4. Dinca M, Fries W, Luisetto G, et al. Evolution of osteopenia in inflammatory bowel disease. Am J Gastroenterol 1999;94:1292-7.

5. Silvennoinen JA, Karttunen TJ, Niemela SE, Manelius JJ, Lehtola JK. A controlled study of bone mineral density in patients with inflammatory bowel disease. Gut 1995;37:71-6.

6. Habtezion A, Silverberg MS, Parkes R, Mikolainis S, Steinhart AH. Risk factors for low bone density in Crohn's disease. Inflamm Bowel Dis 2002;8:87-92. 




The Scientific World Journal
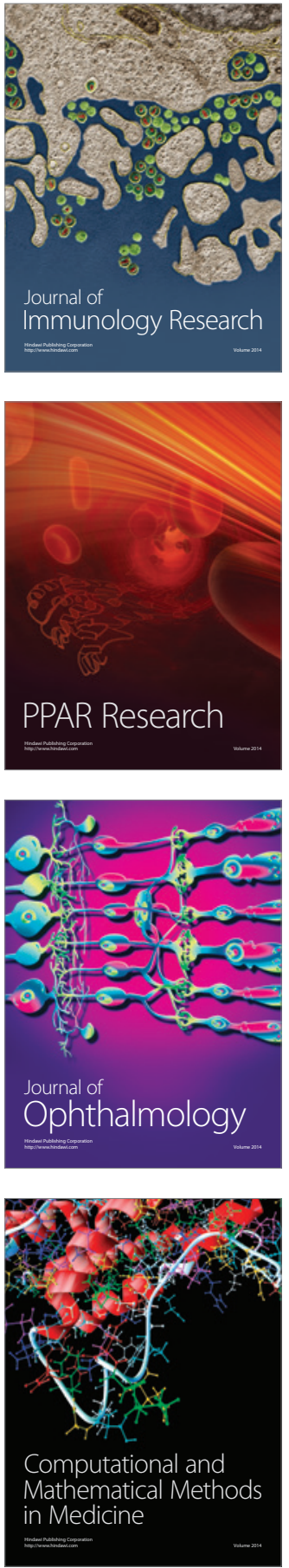

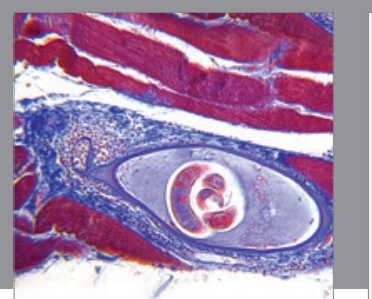

Gastroenterology Research and Practice



\section{Hindawi}

Submit your manuscripts at

http://www.hindawi.com
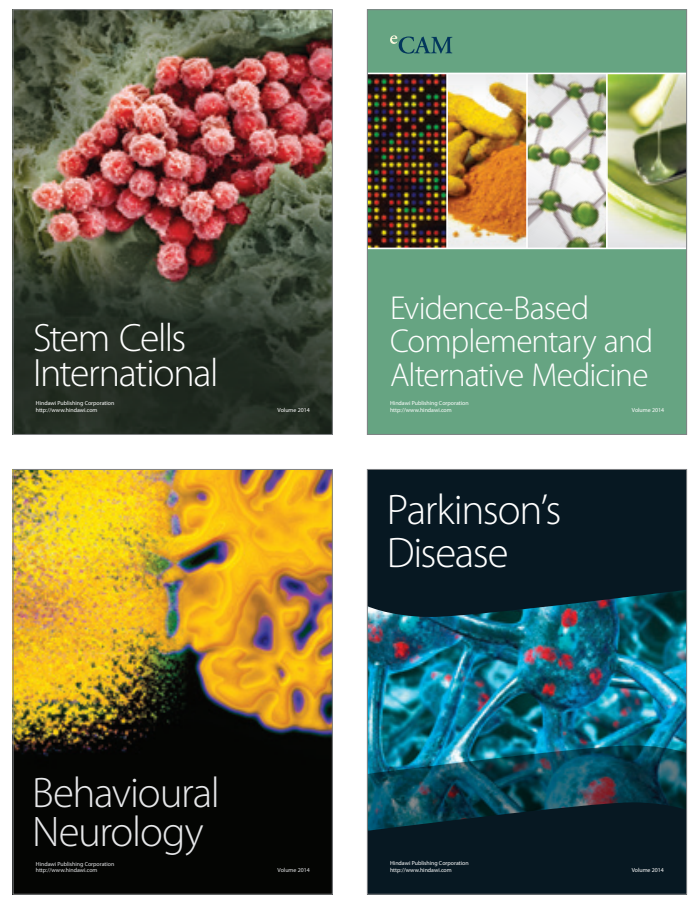
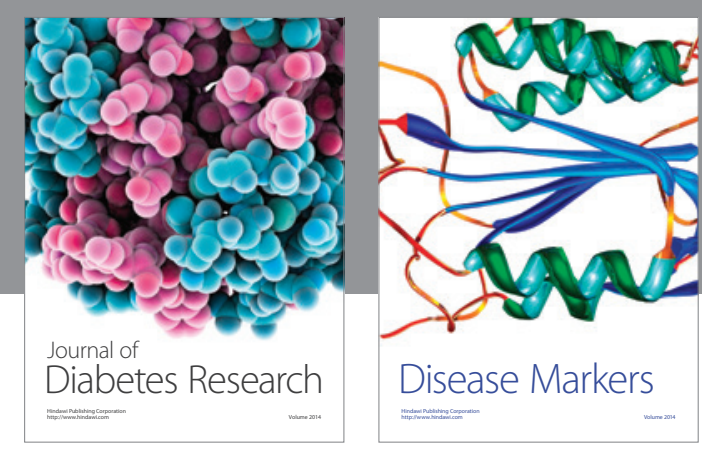

Disease Markers
